\title{
Electron transport in crossed electric and magnetic fields under the condition of the electron streaming in GaN
}

\author{
G.I. Syngayivska and V.V. Korotyeyev \\ V. Lashkaryov Institute of Semiconductor Physics, Department of Theoretical Physics, NAS of Ukraine \\ 41, prospect Nauky,03028Kyiv,Ukraine; e-mail: singg@ukr.net; koroteev@ukr.net
}

\begin{abstract}
High-field electron transport has been studied in crossed electric and magnetic fields in bulk GaN with doping of $10^{16} \mathrm{~cm}^{-3}$ and compensation around $90 \%$ at the low lattice temperature $(30 \mathrm{~K})$. The electron distribution function, the field dependences of the ohmic and Hall components of the drift velocity have been calculated using the Monte Carlo method in the wide range of applied electric $(3 \ldots 15 \mathrm{kV} / \mathrm{cm})$ and magnetic $(1 \ldots 10 \mathrm{~T})$ fields. Two external electrical circuits with short- and open-circuited Hall contacts have been analyzed. For a sample with short-circuited Hall contacts, there are the ranges of magnetic and electric fields where the non-equilibrium electron distribution function has a complicated topological structure in the momentum space with a tendency to formation of the inversion population. For these samples, field dependences of the ohmic and Hall components of the drift velocity have specific character. The ohmic component has the inflection point that corresponds to the maximum point of the Hall component. For the sample with open-circuited Hall contacts, field dependences of the drift velocity demonstrate a sub-linear growth without any critical points. It has been shown that there are ranges of the applied electric and magnetic fields for which the drift velocity exceeds zero magnetic field values.
\end{abstract}

Keywords: Monte Carlo method, gallium nitride, magneto-transport.

Manuscript received 07.10.14; revised version received 04.12.14; accepted for publication 19.02.15; published online 26.02.15.

\section{Introduction}

Semiconductor compounds of group-III nitrides, in particularly $\mathrm{GaN}$, InN and AlN, are perspective materials for the modern high-power and high-frequency micro- and optoelectronics. The strong electron-opticalphonon coupling, large optical phonon energy, sufficiently high electron mobility are inherent for these materials [1-3]. In the literature, nitride materials are widely discussed as candidates for development of the electrical pumping $\mathrm{THz}$ sources, particularly, based on the Gunn effect (under ultra-strong applied electric fields $\approx 100 \mathrm{kV} / \mathrm{cm}$ ) [4-7] as well as streaming transport regime and the optical-phonon transit-time resonance (OPTTR) (under moderate applied electric fields $\approx 2 \ldots 10 \mathrm{kV} / \mathrm{cm}$ ) [8-12]. The streaming transport regime is associated with formation of the strongly-anisotropic distribution function under the sufficiently strong applied electric field. In this case, a dynamic negative differential conductivity can be realized in the specific frequency ranges. Recently, we investigated conditions of streaming and OPTTR effects in the case of compensated $\mathrm{GaN}$, where an undesirable influence of the non-elastic $e-e$ scattering [10] can be avoided. We 
specified intervals of applied electric fields $E$ $(3 \ldots 10 \mathrm{kV} / \mathrm{cm})$, ambient temperatures $T(30 \ldots 150 \mathrm{~K})$ and frequency ranges $(0.2 \ldots 2 \mathrm{THz})$, where streaming and OPTTR effects can occur $[13,14]$. Also, we investigated high-frequency properties of the compensated $\mathrm{GaN}$ under streaming conditions in the case of applied parallel magnetic field $(H \| E)[15,16]$ and found that this field has no effect on steady-state characteristics but essentially changes the frequency spectra of dynamic mobility tensor.

The presence of the external magnetic field, $H$, transversal to the direction of the electric field, $E$, can essentially change dynamics of hot electrons [17] and can effect on the form of the distribution function and current-voltage characteristics. Depending on the ratio of the amplitudes $E$ and $H$, three different transport regimes can be realized. In the weak magnetic fields such as $H<H_{1} \equiv m^{*} c E / P_{0} \quad$ (where $P_{0}=\sqrt{2 m^{*} \hbar \omega_{0}}$ is the electron momentum corresponding to the optical phonon energy $\hbar \omega_{0}, m^{*}$ - electron effective mass, and $c$ - light velocity) electron trajectories in the momentum space are slightly curved, the streaming cyclic motion is maintained, and the electron distribution function remains strongly anisotropic in the direction of the electric field. For intermediate magnetic fields, when $H_{1}<H<H_{2} \equiv 2 H_{1}$, the two electron groups can exists. Electrons of the first group interact with optical phonons and execute the streaming cyclic motion. Electrons of the second group do not interact with optical phonons and move along closed circular orbits in the passive region of the momentum space $\left(|\vec{P}|<P_{0}\right)$. The average energy of electrons belonging to the second group can essentially exceed the thermal energy, $3 / 2 \times k_{\mathrm{B}} T\left(k_{\mathrm{B}}\right.$ is the Boltzmann constant). Therefore, at a sufficiently high electron concentration in the second group the inverted electron distribution can arise, i.e. the distribution function with a double-humped shape in the energy space is formed. In the case of strong magnetic fields, such as $\mathrm{H}>\mathrm{H}_{2}$, trajectories of the most electrons are completely located in the passive region, and the optical phonon emission is strongly suppressed. In this case, the electron distribution function in the momentum space is almost isotropic, and there occurs a collapse of the ohmic current.

The study of the hot electron transport in crossed electric and magnetic fields attracts much attention because of a perspective to obtain inverted distributions necessary for high-frequency generators. However, in conventional $\mathrm{A}^{\mathrm{III}} \mathrm{B}^{\mathrm{V}}$ semiconductor compounds $n$-GaAs, $n$-InSb, $n$-InP these distributions cannot be formed [1820]. The aim of the paper is to study the hot-electron transport in crossed electric and magnetic fields in the novel semiconductor material $\mathrm{GaN}$, in particularly, we consider the effect of magnetic field on the electron distribution function and analyze current-voltage and induction-current characteristics for cases of shortcircuited and open-circuited Hall contacts.

\section{Transport model}

We consider the compensated bulk GaN of cubic modification [21] at the low lattice temperature of $T=$ $30 \mathrm{~K}$ with impurity and electron concentrations of $N_{i}=$ $10^{16} \mathrm{~cm}^{-3}$ and $N_{e}=10^{15} \mathrm{~cm}^{-3}$, respectively. The electron dispersion law is assumed to be parabolic. To calculate electron transport characteristics, the single-particle algorithm of the Monte Carlo procedure [14, 22-24] was applied. In simulation, we assume the electron effective mass, $m^{*}=0.2 m_{e}$ (where $m_{e}$ is the free electron mass) and the optical phonon energy $\hbar \omega_{0}=92 \mathrm{meV}$ [25] and take into account the main three scattering mechanisms: scattering by ionized impurities, acoustic phonons and polar optical phonons. We assume that electric field is applied along the $x$-axis (the direction of the ohmic current) and the magnetic field is applied along the $z$ axis. The $y$-axis is the direction of the Hall current (or field). It should be noted that in all the calculations the magnetic field is treated as a classical field. An estimation shows that for hot electrons with the averaged energies $\bar{\varepsilon} \approx 30 \ldots 50 \mathrm{meV}$ gained within the range of electric fields 3 to $10 \mathrm{kV} / \mathrm{cm}$ the inequality $\hbar \omega_{c}<<\bar{\varepsilon}$ is fulfilled even at magnetic fields of the order of $10 \mathrm{~T}$. Here, $\omega_{c}=e H / m^{*} c$ is the cyclotron frequency with the elementary charge, $e$.

We concentrate to the regions of the magnitudes of $E$ and $H$, where three different transport regimes can be realized (see the diagram in Fig. 1).

In the region (I), the streaming regime is still survived and all electrons reach the energy of the optical phonon. The region (II) corresponds to the case when a portion of electrons has trajectories closed in the passive region. In the region (III), almost all electron trajectories are closed in the passive region. For example, for the electric field $E=5 \mathrm{kV} / \mathrm{cm}$, the region (I) corresponds to magnetic fields $H<1.25 \mathrm{~T}$, region (II) $-1.25 \mathrm{~T}<$ $H<2.5 \mathrm{~T}$ and region (III) $-H>2.5 \mathrm{~T}$.

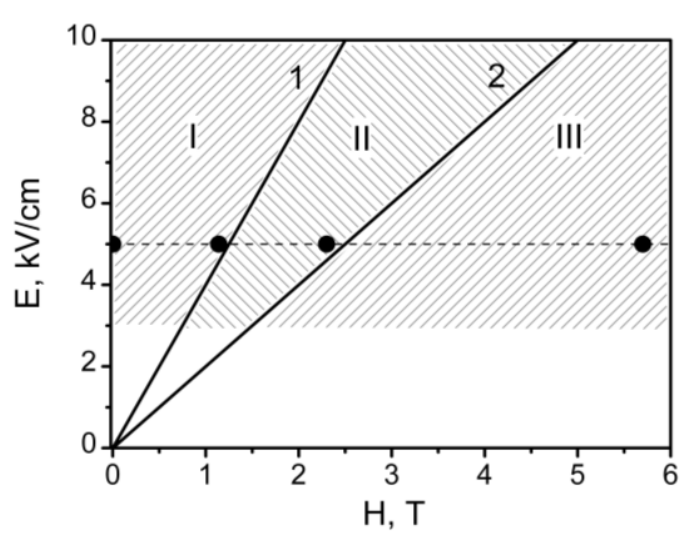

Fig. 1. The $E-H$ plane. Straight lines 1 and 2 correspond to the functions $E=P_{0} H / m^{*} c$ and $E=P_{0} H / 2 m^{*} c$. 


\section{Electron distribution function}

The discussion of the non-equilibrium distribution function is carried out in the energy, $f(\varepsilon)$, and momentum, $f\left(P_{x}, P_{y}\right)$, spaces for the spatially-unlimited sample. Fig. 2 a demonstrates evolution of $f(\varepsilon)$, with variation of the magnetic field at the given electric field $E=5 \mathrm{kV} / \mathrm{cm}$. As seen, in the case of the weak magnetic fields $H \leq 1 \mathrm{~T}$ the streaming distribution is slightly modified: the curve 1 is close to the dash-dotted curve. Note, at this electric field the values of magnetic fields up to $1.2 \mathrm{~T}$ correspond to the region (I) in the diagram (see Fig. 1). In the opposite case of strong magnetic fields corresponding to the region (III), for example at $H=5.7 \mathrm{~T}$, most of electrons move along the trajectories closed in the passive region, and they do not interact with optical phonons. As a result, the distribution function $f(\varepsilon)$ is almost localized in the passive region.
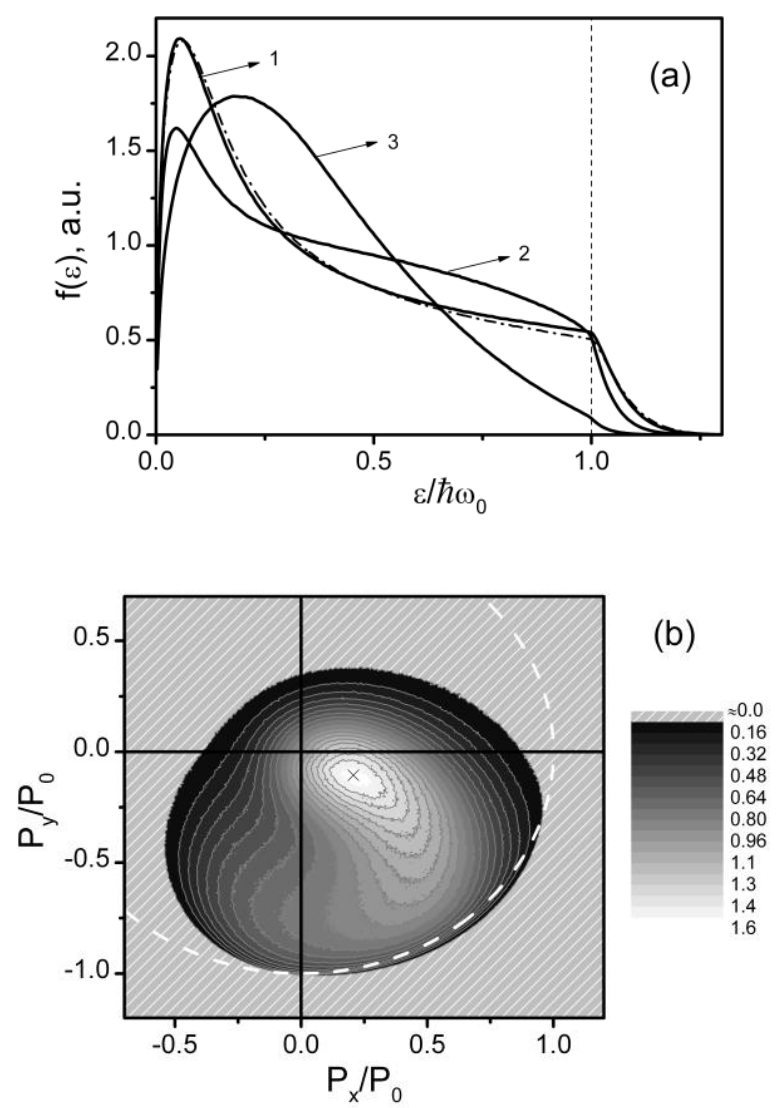

Fig. 2. Panel (a): The electron distribution function $f(\varepsilon)$ for different magnetic fields. Curves $1,2,3$ correspond to $H=1$, $2.3,5.7 \mathrm{~T}$, respectively (these fields are marked by points in Fig. 1). The dash-dotted curve is obtained at $H=0$. Panel (b): The contour plot of the electron distribution function $f\left(P_{x}, P_{y}\right)$ at $H=2.3 \mathrm{~T}$. The dashed circle shows the conditional boundary between passive and active regions. The sign $\times$ denotes the maximum of the distribution. $E=5 \mathrm{kV} / \mathrm{cm}$.
For intermediate magnetic fields corresponding to the region (II), for example $H=2.3 \mathrm{~T}$, it is observed a distinctive reconstruction of the form of distribution function in the energy space. The effect of accumulation of high-energy electrons in the energy interval $\varepsilon / \hbar \omega_{0} \in[0.5-0.9]$ is clear seen. In the momentum space, the distribution function, $f\left(P_{x}, P_{y}\right)$, acquires the complicated topological structure (Fig. 2 b) because of the coexistence of two groups of carriers: electrons executing streaming motion and electrons moving along cyclotron orbits. The maximum of $f\left(P_{x}, P_{y}\right)$ is essentially shifted along both ohmic and Hall directions.

In the works $[20,26]$ for GaAs, it was shown that under weak scattering in the passive region, so that $\omega_{c} \tau / 2 \pi>0.5$ (where $\tau$ is the scattering time in the passive region) it can be formed a double-humped distribution function $f(\varepsilon)$. In our case of $\mathrm{GaN}$ with the impurity concentration of $10^{16} \mathrm{~cm}^{-3}$ at $H=2.3 \mathrm{~T}$ an estimation gives $\tau \approx 0.5 \mathrm{ps}$ and $\omega_{c} \tau / 2 \pi \approx 0.18$. As a result, we observe only weak tendency to formation of this distribution.

In the model case of the very low impurity concentration $\left(N_{i}<10^{14} \mathrm{~cm}^{-3}\right)$, scattering in the passive region is limited by acoustic phonons for which $\tau \approx 50 \mathrm{ps}$ and for magnetic fields corresponding the region (II), for example for $H=2 \mathrm{~T}, \omega_{c} \tau / 2 \pi \approx 18$. As a result, it is observed a well-pronounced separation of the distribution function by two parts. The spindleshaped part corresponds to electrons trapped in the passive region, and the streaming-like part corresponds to electrons interacting with optical phonons (see Fig. 3b). In this case, the distribution function in the energy space has a double-humped shape with an inversion population near the boundary of the passive and active regions (see Fig. 3a).

Thus, in bulk-like GaN samples with $N_{i}=$ $10^{16} \mathrm{~cm}^{-3}$ and $N_{e}=10^{15} \mathrm{~cm}^{-3}$ at $T=30 \mathrm{~K}$, the distribution function with inversion is not formed due to the intensive scattering in the passive region. This distribution can be obtained only in the limit of the small impurity concentration, $N_{i} \approx 10^{14} \mathrm{~cm}^{-3}$.

\section{Transport characteristics}

The transport characteristics in the magnetic field depend on the choice of configuration of the external electrical circuit. There are two typical schemes of measurements: 1) scheme of short-circuited Hall contacts and 2) scheme of open-circuited Hall contacts. The first scheme also can be realized for the short sample in the direction of the electric field, and the second scheme can be realized for the long sample in the direction of the electric field. Calculations of transport characteristics were carried out for both schemes. 

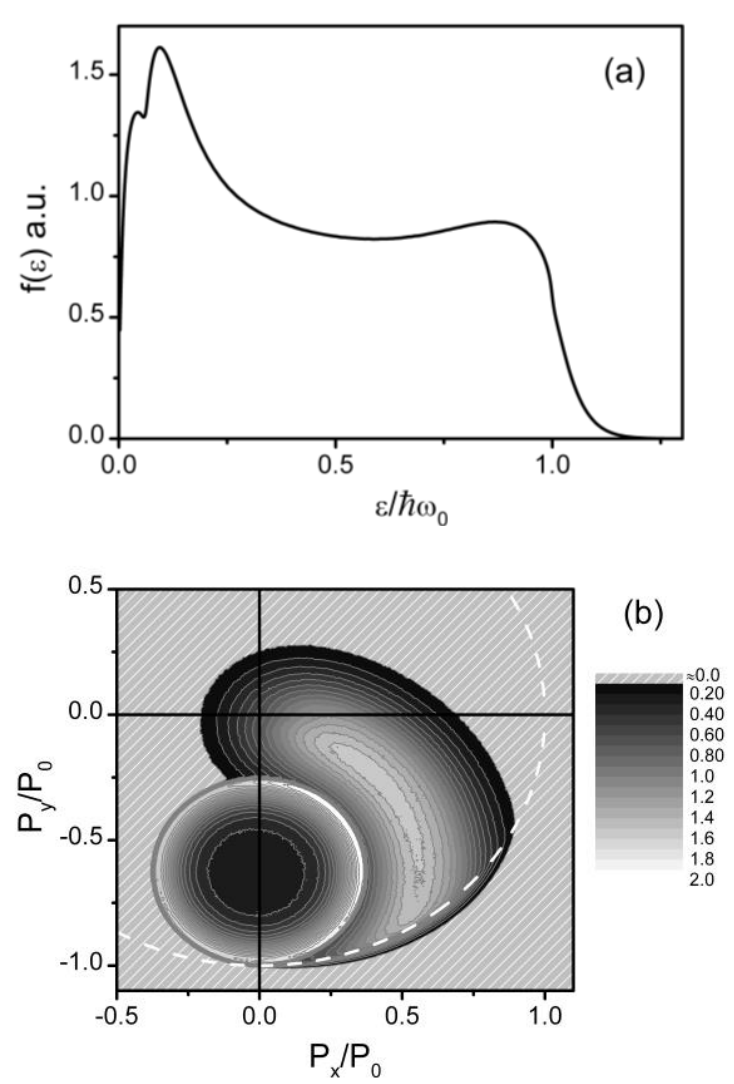

Fig. 3. Panel (a): The electron distribution in the energy space. Panel (b): The contour plot of the electron distribution function $f\left(P_{x}, P_{y}\right)$ in the momentum space. For both panels, $E=$ $5 \mathrm{kV} / \mathrm{cm}$ and $H=2 \mathrm{~T}$.

\subsection{Short-circuited Hall contacts}

For the case of short-circuited Hall contacts, the effect of the charge accumulation at Hall edges can be neglected, and the Hall field, $E_{h}$, is equal to zero. In the magnetic field, the Lorentz force deviates electrons to the $y$ direction, which results in appearance of the Hall component of the drift velocity, $V_{h}$, transversal to the applied electric field $E$. Thus, the vector of the drift velocity $\vec{V}_{\text {tot }}$ subtends an angle $\theta$ (the Hall angle) with the vector of the applied electric field $\vec{E}$. The angle $\theta$ can be determined as follows: $\tan \theta=V_{h} / V_{d}$, where $V_{d}$ is the ohmic component of the drift velocity, which is directed along $\vec{E}$. The Monte Carlo procedure allows to calculate the components $V_{h}$ and $V_{d}$ and angle $\theta$.

Typical magnetic and electric fields dependences of both ohmic and Hall components of the drift velocity are shown in Figs. $4 \mathrm{a}$ and $4 \mathrm{~b}$, respectively.

As seen, functions $V_{d}(H)$ and $V_{h}(H)$ consist of three portions with a distinct behavior. Let us consider the pair of curves calculated for $5 \mathrm{kV} / \mathrm{cm}$. In magnetic fields up to $\approx 1 \mathrm{~T}, V_{d}(H)$ is weakly changed, and $V_{h}(H)$ exhibits a linear behavior. Starting from the field $1.25 \mathrm{~T}$, when accumulation of electrons in the passive region is activated, $V_{d}(H)$ is rapidly decreased with increasing the magnetic field. The inflection point in the dependence of $V_{d}(H)$ corresponds to the maximum point in the dependence of $V_{h}(H)$. In magnetic fields $H>2.5 \mathrm{~T}$ (the characteristic field $\mathrm{H}_{2}$ ), both characteristics show a gradually decreasing behavior. In the range of high magnetic fields $H>5 \mathrm{~T}$, the electron drift is almost suppressed along $E$ (the collapse of the ohmic current) but it is still remained along the Hall direction. With increasing the applied electric field, both characteristic magnetic fields $H_{1}$ and $H_{2}$ are increased, too, therefore it is observed the general broadening of magnetic field dependences with shifting the inflection and maximum points to the range of stronger magnetic fields (see thin lines in Fig. 4a).
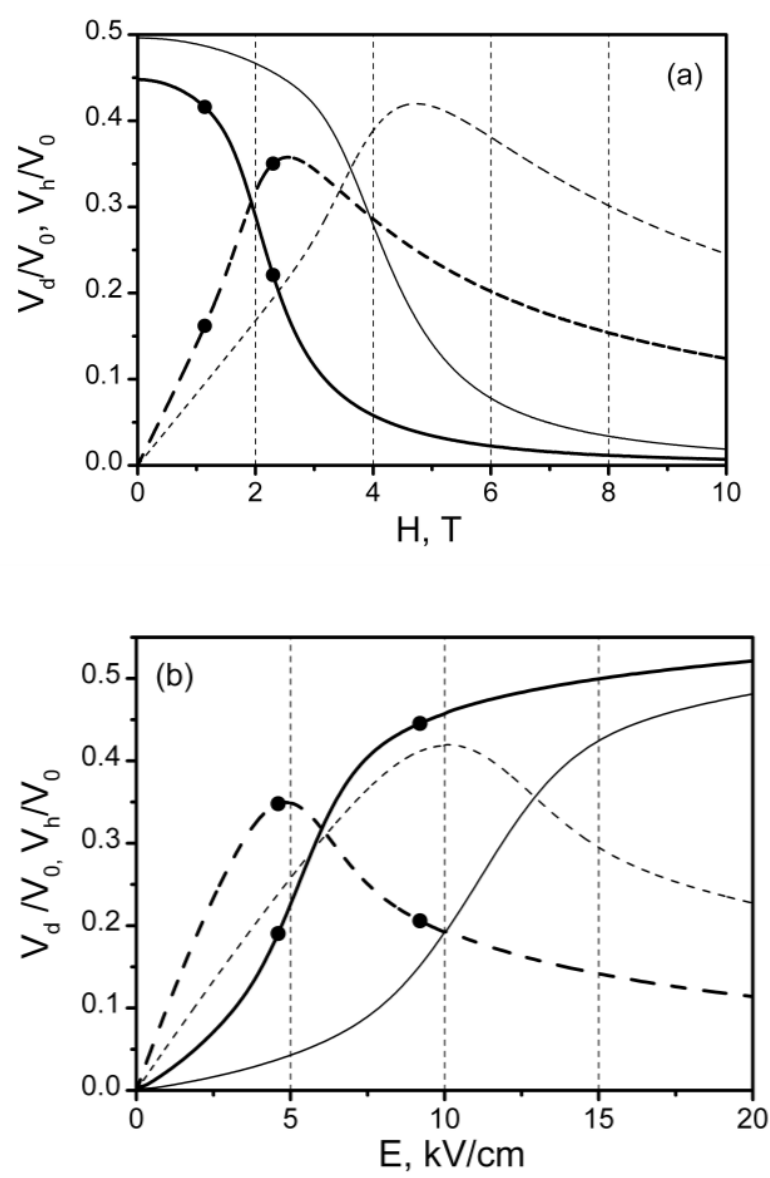

Fig. 4. Panel (a): Dependences of $V_{d}(H)$ (solid lines) and $V_{h}(H)$ (dashed lines) at $E=5 \mathrm{kV} / \mathrm{cm}$ (thick lines) and $E=$ $10 \mathrm{kV} / \mathrm{cm}$ (thin lines). Points depict characteristic magnetic fields $H_{1}=1.14 \mathrm{~T}$ and $H_{2}=2.3 \mathrm{~T}$. Panel (b): Dependences of $V_{d}(E)$ (solid lines) and $V_{h}(E)$ (dashed lines) at $H=2.3 \mathrm{~T}$ (thick lines) and $H=4.6 \mathrm{~T}$ (thin lines). Points mark characteristic electric fields $E=4.6,9.2 \mathrm{kV} / \mathrm{cm}$, corresponding to magnetic fields $H_{1}$ and $H_{2}$. The velocity $V_{0}=4 \cdot 10^{7} \mathrm{~cm} / \mathrm{s}$. 

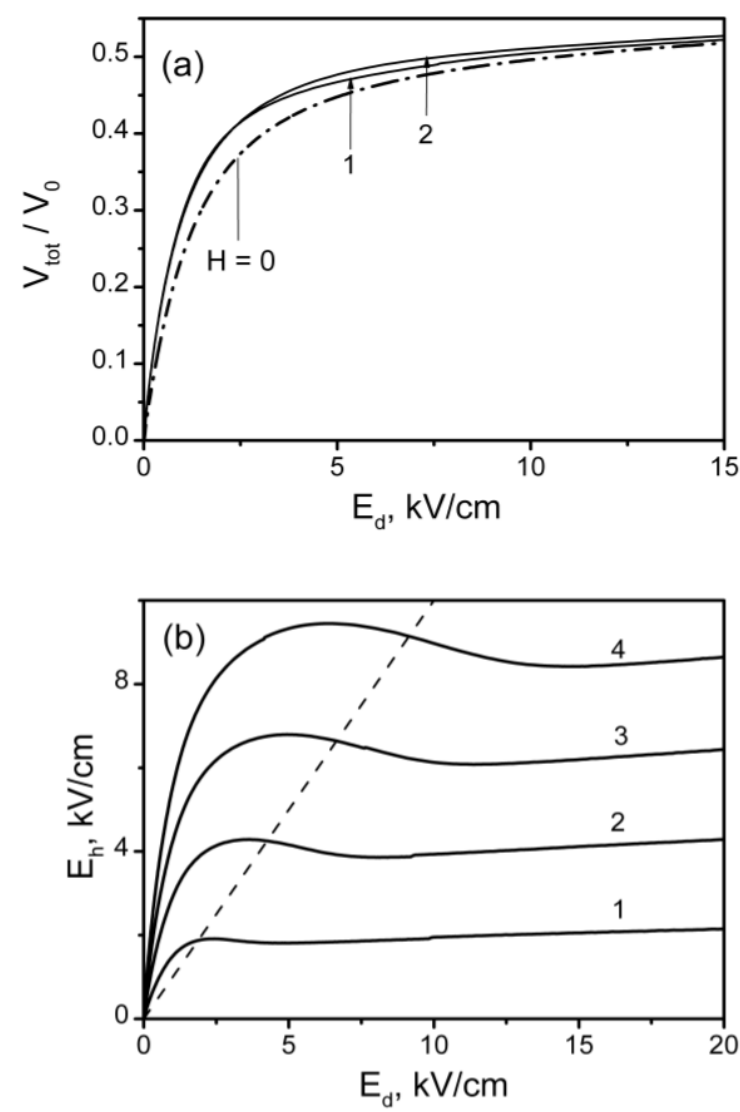

Fig. 5. Panel (a): Field dependences of the drift velocity at $H=$ 3.4 and $5.7 \mathrm{~T}$ (curves 1 and 2, respectively). The dash-dotted curve is calculated for $H=0$. Panel (b): Dependences of $E_{h}\left(E_{d}\right)$ for the magnetic fields $H=1.14,2.3,3.4,4.6 \mathrm{~T}$ (curves $1,2,3$ and 4 , respectively). The dashed straight line crosses curves $1-4$ at points where $E_{h}$ is equal to $E_{d}$.

Electric field characteristics $V_{d}(E)$ and $V_{h}(E)$ also have three specific intervals. At $H=2.3 \mathrm{~T}$ (see thick lines in Fig. 4b), a super-linear growth of $V_{d}(E)$ and linear growth of $V_{h}(E)$ are observed in the interval $0 \ldots .5 \mathrm{kV} / \mathrm{cm}$ that corresponds to the region (III) in the diagram (Fig. 1). The inflection point in the dependence of $V_{d}(E)$ corresponds to the maximum point in the dependence of $V_{h}(E)$. In the range of electric fields corresponding to the region (II), the ohmic component shows a sub-linear behavior with tending to the quasisaturation at higher electric fields belonging to the region (I). In this case, the Hall component is essentially decreased within the range $E=5 \ldots 9 \mathrm{kV} / \mathrm{cm}$. At $E$ > $10 \mathrm{kV} / \mathrm{cm}$, it is observed a slow dropping behavior of $V_{h}(E)$. With increasing the applied magnetic field, the inflection and maximum points are shifted to the range of stronger electric fields.

It should be noted that dependences of $V_{d}$ and $V_{h}$ have the crossing point (at which the Hall angle is equal to $\pi / 4$ ). Thus, there are ranges of $E$ and $H$ where one of drift velocity components dominates.

\subsection{Open-circuited Hall contacts}

For the case of the sample with open-circuited Hall contacts (an open-circuited sample), the Hall electric field, $E_{h}$, is not equal to zero due to the charge accumulation on Hall edges, and the Hall current is absent. For this external circuit, it is usually measured a total current flowing through the sample, and the Hall voltage as functions of the applied field $E_{d}$. If the sample is so long in the Hall direction that spatial inhomogeneity of the electric field near the Hall contacts can be neglected, then field dependences of the drift velocity, $V_{t o t}\left(E_{d}\right)$, and Hall field, $E_{h}\left(E_{d}\right)$, can be easy obtained using results for the case of a sample with short-circuited Hall contacts (a short-circuited sample). From simple geometrical considerations, it follows: $V_{\text {tot }}=\sqrt{V_{d}^{2}+V_{h}^{2}}, \quad E_{d}=E \cos \theta, \quad E_{h}=E \sin \theta$. For the open-circuited sample, dependences of $V_{\text {tot }}\left(E_{d}\right)$ (Fig. 5a) have monotonic behavior, and in contrast to the short-circuited sample, there are no inflection points. Note that the drift velocity under applied magnetic fields can exceed its value at zero magnetic field. The similar result was obtained and discussed in refs. [27, 28] relating to $n$-GaAs and $n$-InP samples.

Fig. $5 \mathrm{~b}$ demonstrates dependences of $E_{h}\left(E_{d}\right)$ for several values of magnetic fields. All dependences have similar behavior: in electric and magnetic fields corresponding to the region (III), the Hall field exhibits rapid growth to the maximum. Then, for the applied fields corresponding to the region (II) the Hall field smoothly decreases and remains almost constant at applied fields belonging to the region (I).

\section{Conclusions}

The high-field electron transport characteristics in crossed electric and magnetic fields are studied for the case of the compensated bulk-like GaN. In the frames of numerical solution of Boltzmann transport equation by using the Monte Carlo method, it has been calculated distribution functions in the energy and momentum spaces, electric and magnetic field dependences of the ohmic and Hall components of the drift velocity.

It has been analyzed two cases of the external Hall circuit. The first one is the case of the sample with shortcircuited Hall contacts, and the second one is the case of the sample with open-circuited Hall contacts.

For the short-circuited sample, we determined intervals of applied electric and magnetic fields that correspond to different regimes of the electron transport. It has been found that in weak magnetic fields $H<H_{1}$, for example in $H<1 \mathrm{~T}$ at $E=5 \mathrm{kV} / \mathrm{cm}$, the streaming transport regime is kept. In this case, the ohmic component of the drift velocity is weakly, and the Hall component shows a linear behavior. In the range of the magnetic fields $H_{1}<H<H_{2}=2 H_{1}$, for example in $H=$ $1 \ldots 3 \mathrm{~T}$ at $E=5 \mathrm{kV} / \mathrm{cm}$, the streaming regime is 
destroyed, and the distribution function acquires the complicated topological structure conditioned by the coexistence of two groups of electrons: electrons of the first group execute the cyclic motion typical for the streaming, and electrons of the second group execute the cyclotron motion without interaction with optical phonons. In this range of magnetic fields, it is observed rapid decreasing the ohmic component, and the characteristic inflection point; the Hall component is increased and reaches the maximum. The position of inflection and maximum points correspond to the magnetic field $H \approx H_{2}(H \approx 2.5 \mathrm{~T}$ for $E=5 \mathrm{kV} / \mathrm{cm})$. In the magnetic fields $H \approx 5 \mathrm{~T}$, almost all electrons execute the cyclotron motion and do not interact with optical phonons. As a result, the Hall current is essentially larger than the ohmic one. With increasing the applied electric field, the style of $H$-dependences for both components of the drift velocity is kept, but it is observed the broadening of dependences (inflection and maximum points correspond to larger magnetic fields).

The ohmic component of the drift velocity vs $E$ is the rising characteristic in contrast to its dropping dependence vs $H$. Styles of $E$ - and $H$-dependences of the Hall component are similar. $E$-dependences of the drift velocity also contain inflection (for the ohmic component) and maximum (for the Hall component) points. With increasing the applied magnetic field, $E$ dependences are changed in the similar way to those of $H$-dependences.

For the open-circuited sample, the drift velocity has only the ohmic component, but the electric field has two non-zero ohmic and Hall components. The drift velocity as a function of the applied electric field is the rising characteristic, and it does not show the peculiarities inherent for the short-circuited sample (the inflection point is absent). But at the given magnetic fields, there is the interval of negative magnetoresistance, i.e. the values of the drift velocity exceed those in zero magnetic field.

It has been demonstrated that in the presence of the magnetic field the current-voltage characteristics in the ohmic direction obtained for samples with short- and open-circuited Hall contacts are dramatically differed.

\section{References}

1. M.J. Manfra, N.G. Weimann, J.W.P. Hsu et al., High mobility AIGaN/GaN heterostructures grown by plasma-assisted molecular beam epitaxy on semi-insulating GaN templates prepared by hydride vapor phase epitaxy // J. Appl. Phys. 92, p. 338-345 (2002).

2. D.C. Look and J.R. Sizelove, Predicted maximum mobility in bulk GaN // Appl. Phys. Lett. 79(8), p. 1133-1135 (2001).

3. L. Bouguen, S. Contreras, A.B. Jouault, L. Konczewicz, J. Camassel, Y. Cordier, M. Azize, S. Chenot and $\mathrm{N}$. Baron, Investigation of $\mathrm{AlGaN} / \mathrm{AlN} / \mathrm{GaN}$ heterostructures for magnetic sensor application from liquid helium temperature to $300{ }^{\circ} \mathrm{C} / / \mathrm{Appl}$. Phys. Lett. 92, 043504 (2008).

4. V.N. Sokolov, K.W. Kim, V.A. Kochelap and D.L. Woolard, High-frequency small-signal conductivity of hot electrons in nitride semiconductors // Appl. Phys. Lett. 84(18), p. 36303632 (2004); Terahertz generation in submicron $\mathrm{GaN}$ diodes within the limited space-charge accumulation regime // J. Appl. Phys. 98(6), 064507 (2005).

5. B.A. Danilchenko, S.E. Zelensky, E. Drok, S.A. Vitusevich, S.V. Danylyuk, N. Klein, H. Luth, A.E. Belyaev and V.A. Kochelap, Hot-electron transport in $\mathrm{AlGaN} / \mathrm{GaN}$ two-dimensional conducting channels // Appl. Phys. Lett. 85 (22), p. 5421-5423 (2004)

6. S.A. Vitusevich, S.V. Danylyuk, N. Klein, M.V. Petrychuk, V. N. Sokolov, V.A. Kochelap, A.E. Belyaev, V. Tilak, J. Smart, A. Vertiatchikh, Excess low-frequency noise in $\mathrm{AlGaN} / \mathrm{GaN}$-based high-electron-mobility transistors // Appl. Phys. Lett. 80(12) p. 2126-2128 (2002).

7. O. Yilmazoglu, K. Mutamba, D. Pavlidis and T. Karaduman, Measured negative differential resistivity for GaN Gunn diodes on $\mathrm{GaN}$ substrate // Electronics Lett. 43(8), p. 480-482 (2007).

8. E.A. Barry, K.W. Kim, and V.A. Kochelap, Hot electrons in group-III nitrides at moderate electric fields // Appl. Phys. Lett. 80, p. 2317-2319 (2002).

9. E. Starikov, P. Shiktorov, V. Gruzinskis, L. Varani, C. Palermo, J-F Millithaler and L. Regiani, Frequency limits of terahertz radiation generated by optical-phonon transit-time resonance in quantum wells and heterolayers // Phys. Rev. B, 76, 045333 (2007); Terahertz generation in nitrides due to transit-time resonance assisted by optical phonon emission // J. Phys.: Condens. Matter, 20, 384209 (2008).

10. J.T. Lu and J.C. Cao, Monte Carlo study of terahertz generation from streaming distribution of two-dimensional electrons in a $\mathrm{GaN}$ quantum well // Semicond. Sci. Technol., 20, p. 829-833 (2005).

11. V.V. Korotyeyev, V.A. Kochelap, K.W. Kim, and D.L.Woolard, Streaming distribution of twodimensional electrons in III-N heterostructures for electrically pumped terahertz generation // Appl. Phys. Lett. 82, p. 2643-2645 (2003).

12. K.W. Kim, V.V. Korotyeyev, V.A. Kochelap, A.A. Klimov, and D.L. Woolard, Tunable terahertz-frequency resonances and negative dynamic conductivity of two-dimensional electrons in group-III nitrides // J. Appl. Phys. 96, p. 6488-6491 (2004).

13. G.I. Syngayivska and V.V. Korotyeyev, Monte Carlo simulation of hot electron effects in compensated $\mathrm{GaN}$ semiconductor at moderate electric fields // Semiconductor Physics, Quantum Electronics \& Optoelectronics, 10(4), p. 54-59 (2007). 
14. G.I. Syngayivska, V.V. Korotyeyev, Electrical and high-frequency properties of compensated $\mathrm{GaN}$ under electron streaming conditions // Ukr. J. Phys. 58(1), p. 40-55 (2013).

15. G.I. Syngayivska, V.V. Korotyeyev and V.A. Kochelap, High-frequency response of $\mathrm{GaN}$ in moderate electric and magnetic fields: Interplay between cyclotron and optical phonon transient time resonances // Semicond. Sci. Technol. 28, 035007 (2013).

16. V.V. Korotyeyev, Peculiarities of THzelectromagnetic wave transmission through the $\mathrm{GaN}$ films under conditions of cyclotron and optical phonon transit-time resonances // Semiconductor Physics, Quantum Electronics \& Optoelectronics, 16(1), p. 18-26 (2013).

17. E. Vosilyus and E. Levinson, Optical phonon production and galvanomagnetic effects for a largeanisotropy electron distribution // Zhurnal Eksperiment. Teor. Fiziki, 50, p. 1660-1666 (1966), in Russian; Galvanomagnetic effects in strong electric fields during nonelastic electron scattering // Zhurnal Eksperiment. Teor. Fiziki, 52, p. 10131024 (1967), in Russian.

18. I.B. Levinson, Transport phenomena in systems with pronounced dynamics in crossed fields, in: Hot Electrons in Semiconductors: Streaming and Anisotropic Distributions in Crossed Fields, Eds. A.A. Andronov and Yu.K. Pozela. Gorki, 1983, p. 82-100 (in Russian).

19. V.A. Valov, V.A. Kozlov, L.S. Mazov and I.M. Nefedov, Anisotropic and inverted hot carrier distributions in n-InSb, n-GaAs and p-Ge in crossed E- and H-fields, in: Inverted Distributions of Hot Electrons in Semiconductors, Eds. A.A. Andronov and Yu.K. Pozela. Gorki, 1983, p. 17-55 (in Russian).

20. R.S. Brazis, E.V. Starikov and P.N. Shiktorov, Two groups of electrons in crossed fields for lowtemperature optical scattering, in: Hot Electrons in Semiconductors: Streaming and Anisotropic
Distributions in Crossed Fields, Eds. A.A. Andronov and Yu.K. Pozela. Gorki, 1983, p. 114-150 (in Russian).

21. O. Brandt, H. Yang, J.R. Mullhauser, A. Trampert and K.H. Ploog, Properties of cubic GaN grown by MBE // Mater. Sci. Eng. B, 43, p. 215-221 (1997).

22. A.D. Boardmann, W. Fawcett and S. Swain, Monte Carlo determination of electron transport in gallium arsenide // J. Phys. Chem. Solids, 31, p. 1963-1990 (1970).

23. A.D. Boardmann, W. Fawcett and J.G. Ruch, Monte Carlo determination of hot electron galvanomagnetic effects in gallium arsenide // phys. status solidi (a), 4, p. 133-141 (1971).

24. C. Jacoboni and L. Reggiani, The Monte Carlo method for the solution of charge transport in semiconductors with applications to covalent materials // Rev. Mod. Phys. 55(3), p. 645-705 (1983).

25. M. Levinstein, S. Rumyantsev, and M. Shur, Properties of Advanced Semiconductor Materials: GaN, AlN, InN, BN, SiC, SiGe. Wiley, New York, 2001.

26. Ya.I. Al'ber, A.A. Andronov, V.A. Valov, V.A. Kozlov, A.M. Lerner and I.P. Ryazantseva, Inverted hot-electron states and negative conductivity in semiconductors // Zhurnal Eksperiment. Teor. Fiziki, 72(3), p. 1030-1050 (1977), in Russian [Sov. Phys. JETP, 45(3), p. 539-550 (1977)].

27. S. Kachyulis, I. Parshyalyunas, G. Tamulaitis, Electric conductivity in $\mathrm{n}-\mathrm{GaAs}$ and $\mathrm{n}-\mathrm{InP}$ in crossed electric and magnetic fields at $T=77 \mathrm{~K}$, in: Hot Electrons in Semiconductors: Streaming and Anisotropic Distributions in Crossed Fields, Eds. A.A. Andronov and Yu.K. Pozela. Gorki, 1983, p. 101-113 (in Russian).

28. E.M. Gershenzon, L.B. Litvak-Gorskaya, R.I. Rabinovich and E.Z. Shapiro, Cooling of nonequilibrium electrons and negative magnetoresistance in strong electric and magnetic fields // Zhurnal Eksperiment. Teor. Fiziki, 90, p. 248-258 (1986), in Russian [Sov. Phys. JETP, 63(1), p. 142148 (1986)]. 